November 23, 2008

\title{
Skyrmions at finite density and temperature: the chiral phase transition
}

\author{
Byung-Yoon Park ${ }^{(a)}$, Hee-Jung Lee ${ }^{(b)}$, and Vicente Vento ${ }^{(c)}$ \\ (a) Department of Physics, Chungnam National University, Daejon 305-764, Korea \\ (E-mail: bypark@cnu.ac.kr) \\ (b) School of Science Education, Chungbuk National University, Cheongju Chungbuk 361-763, \\ Korea \\ (E-mail: hjl@chungbuk.ac.kr) \\ (c) Departament de Fisica Tè̀rica and Institut de Física Corpuscular \\ Universitat de València and Consejo Superior de Investigaciones Científicas \\ E-46100 Burjassot (València), Spain
}

(E-mail: Vicente.Vento@uv.es)

\begin{abstract}
The Skyrme model, an effective low energy theory rooted in large $N_{c}$ QCD, has been applied to the study of dense matter. Matter is described by various crystal structures of skyrmions. When this system is heated, the dominating thermal degrees of freedom are the fluctuating pions. Taking these mechanisms jointly produces a description of the chiral phase transition leading to the conventional phase diagram with critical temperatures and densities in agreement with expected values.
\end{abstract}

Pacs: 11.30.Rd, 12.39.Dc, 14.40.Aq, 25.75.Nq

Keywords: skyrmion, dense matter, finite temperature, fluctuating pions, chiral phase transition 
An important issue today is to understand the properties of hadronic matter under extreme conditions, e.g., at high temperature as in relativistic heavy-ion physics and/or at high density as in compact stars. The phase diagram of hadronic matter turns out richer than what has been predicted by perturbative Quantum Chromodynamics (QCD) [1]. Lattice QCD, the main computational tool accessible to highly nonperturbative QCD, has provided much information on the the finite temperature transition, such as the value of the critical temperature, the type of equation of state, etc 2. However, due to a notorious 'sign problem', lattice QCD could not be applied to study dense matter. Only in the last few years, it has become possible to simulate QCD with small baryon density [3]. Chiral symmetry is a flavor symmetry of QCD which plays an essential role in hadronic physics. At low temperatures and densities it is spontaneously broken leading to the existence of the pion. Lattice studies seem to imply that chiral symmetry is restored in the high temperature and/or high baryon density phases and that it may go handin-hand with the confinement/deconfinement transition. The quark condensate $\langle\bar{q} q\rangle$ of QCD is an order parameter of this symmetry and decreases to zero when the symmetry is restored.

The Skyrme model, an effective low energy theory rooted in large $N_{c}$ QCD, has been applied to the dense matter studies [4, 5, 6, 7, 8, 9, 10, The model does not have explicit quark and gluon degrees of freedom, and therefore one can not investigate the confinement/deconfinement transition directly, but we may study the chiral symmetry restoration transition which occurs close by. The main ingredient associated with chiral symmetry is the pion, the Goldstone boson associated with the spontaneously broken phase. The various patterns in which the symmetry is realized in QCD will be directly reflected in the in-medium properties of the pion and consequently in the properties of the skyrmions made of it.

The classical nature of skyrmions enables us to construct the dense system quite conveniently by putting more and more skyrmions into a given volume. Then, skyrmions shape and arrange themselves to minimize the energy of the system. The ground state configuration of skyrmion matter are crystals. At low density it is made of well-localized single skyrmions [11. At a critical density, the system undergoes a structural phase transition to a new kind of crystal. It is made of 'half-skyrmions' which are still well-localized but carry only half winding number. In the half-skyrmion phase, the system develops an additional symmetry which leads to a vanishing average value for $\langle\sigma\rangle$ [12]. In the studies of the late 80's [13], the vanishing of $\langle\sigma\rangle$ was interpreted as chiral symmetry restoration by assuming that $\langle\sigma\rangle$ is related to the QCD order parameter $\langle\bar{q} q\rangle$. However, in Ref. [4], it was shown that the vanishing of $\langle\sigma\rangle$ cannot be an indication of a genuine chiral symmetry restoration, because the decay constant of the pion fluctuating in such a halfskyrmion matter does not vanish. This was interpreted as a signal of the appearance of a pseudo-gap phase similar to what happens in high $T_{c}$ superconductors [14].

The puzzle was solved in Ref. [7] by incorporating a suitable degree of freedom, the dilaton field $\chi$, associated to the scale anomaly of QCD. The dilaton field takes over the role of the order parameter for chiral symmetry restoration. As the density of skyrmion matter increases, both $\langle\sigma\rangle$ and $\langle\chi\rangle$ vanish (not necessarily at the same critical density). The effective decay constant of the pion fluctuation vanishes only when $\langle\chi\rangle$ becomes zero. It is thus the dilaton field which provides the mechanism for chiral symmetry restoration.

Contrary to lattice QCD, there are few studies on the temperature dependence of skyrmion matter. Skyrmion matter has been heated up to melt the crystal into a liquid to investigate the crystal-liquid phase transition [15, 16] a phenomenon which is irrelevant to the restoration of chiral symmetry. In here, we study skyrmion matter at finite density and temperature. We obtain the phase diagram describing the realization of the chiral symmetry.

The basic strategy of our approach is as follows. We describe dense skyrmion matter as a certain crystal structure. Thereafter, we let the temperature rise. The dominating thermal 
degrees of freedom in this process are the fluctuating pions which contribute a thermal energy proportional to $T^{4}$. We neglect the thermal fluctuations of the heavy skymions (translations, rotations and vibrations) which are proportional to $T$. We show that this strategy leads to a familiar phase diagram with critical temperature and density in agreement with the expected values.

The simplest model Lagrangian containing the inevitable ingredients to make the calculation respect the flavor properties of $Q C D$ reads [17, 18]

$$
\mathcal{L}=\chi^{2} \mathcal{L}_{\sigma}+\mathcal{L}_{\mathrm{sk}}-\mathcal{V}(\chi)
$$

with

$$
\begin{gathered}
\mathcal{L}_{\sigma}=\frac{f_{\pi}^{2}}{4} \operatorname{tr}\left(\partial^{\mu} U^{\dagger} \partial_{\mu} U\right), \\
\mathcal{L}_{\mathrm{sk}}=\frac{1}{32 e_{\mathrm{sk}}^{2}} \operatorname{tr}\left[U^{\dagger} \partial_{\mu} U, U^{\dagger} \partial_{\nu}\right]^{2} \\
\mathcal{V}(\chi)=\frac{m_{\chi}^{2} f_{\chi}^{2}}{4}\left\{\chi^{4}\left(\ln \chi-\frac{1}{4}\right)+\frac{1}{4}\right\} .
\end{gathered}
$$

In this equation, $U=\exp \left(i \vec{\tau} \cdot \vec{\pi} / f_{\pi}\right)(\in S U(2))$ is a nonlinear realization of the pion fields and $\chi$ represents the dilaton field. The model Lagrangian contains four parameters, $f_{\pi}, e_{\mathrm{sk}}, m_{\chi}$ and $f_{\chi}$, i.e. the pion decay constant, the Skyrme parameter, the dilaton decay constant and the dilaton mass, respectively. We choose in what follows $f_{\pi}=93 \mathrm{MeV}, e_{\mathrm{sk}}=4.75, m_{\chi}=720$ $\mathrm{MeV}$ and $f_{\chi}=210 \mathrm{MeV}$ (a discussion on the values of these parameters can be found in Ref. [7]). For simplicity we neglect the pion mass and take the $\chi$ field as a constant in space and time. Still, the 'constant' dilaton field controls the chiral phase transition through the potential energy term. In the vacuum, this term has its minimum at $\chi=1$. At finite density and temperature, skyrmions and thermal pions contribute to the term proportional to $\chi^{2}$ and the system undergoes a dynamical phase transition when this term is larger than the potential energy. While the specific values for the critical temperature and density depend strongly on the chosen parameters, the phase transition scenario remains qualitatively the same for all reasonable values.

Skyrmion matter is described by the energy of a single skyrmion:

$$
\mathcal{E}(\rho)=\chi^{2} \mathcal{E}_{\sigma}+\mathcal{E}_{\mathrm{sk}}+\mathcal{V}(\chi) V
$$

where

$$
\begin{gathered}
\mathcal{E}_{\sigma}(\rho)=\int_{V} d^{3} r \operatorname{tr}\left(\partial_{i} U_{0} \partial_{i} U_{0}^{\dagger}\right), \\
\mathcal{E}_{\text {sk }}(\rho)=\int_{V} d^{3} r \operatorname{tr}\left[U_{0}^{\dagger} \partial_{i} U_{0}, U_{0}^{\dagger} \partial_{j} U_{0}\right]^{2},
\end{gathered}
$$

$\rho$ is the baryon number density of the system and $V$ is the volume occupied by a single skyrmion; i.e., $\rho=1 / V . U_{0}(\vec{r})$ is the static skyrmion configuration that minimizes the system energy $\mathcal{E}$ for a given $\rho$. Note that both the potential energy terms, which contains an explicit $1 / \rho(=V)$ factor, and $\mathcal{E}_{\sigma, \text { sk }}$, through $U_{0}(\vec{r})$, depend on the baryon number density.

What happens if we heat up the system? Naively, as the temperature increases, the kinetic energy of the skyrmions increases and the skyrmion crystal begins to melt. The kinetic energy associated with the translations, vibrations and rotations of the skyrmions is proportional to $T$. This mechanism leads to a solid-liquid-gas phase transition of the skyrmion system. However, we are not interested in this transition but in the chiral symmetry restoration transition. A new 
mechanism must be incorporated to describe it and it happens to be the thermal excitation of the pions in the medium. This phenomenon is proportional to $T^{4}$ and therefore dominates the absorption of heat. We only consider the latter in our discussion.

The pressure of the non-interacting pions is given by [19]

$$
P=\frac{\pi^{2}}{30} T^{4}
$$

where we have taken into account the contributions from three pions, $\pi^{+}, \pi^{0}, \pi^{-}$. This term contributes to the energy per single skyrmion volume as $3 P V \chi^{2}$. The factor 3 comes from the fact that our pions are massless. The kinetic energy of the pions arises from $\mathcal{L}_{\sigma}$, and therefore scaling implies that it should carry a factor $\chi^{2}$. After including the thermal pions, the energy lodged around a single skyrmion can be expressed as

$$
\mathcal{E}(\rho, T)=\left(\mathcal{E}_{\sigma}(\rho)+\frac{\pi^{2}}{10} T^{4} V\right) \chi^{2}+\mathcal{E}_{\text {sk }}(\rho)+\mathcal{V}(\chi) V .
$$

In order to complete our calculation we have to determine the values of $U_{0}(\vec{r})$ and $\chi$ that minimize $\mathcal{E}(\rho, T)$ for a given density and temperature.

Chiral restoration will occur when $\chi$ vanishes. How does this take place in hot and dense matter? In eq.(4), the potential energy of the dilaton is finite and its magnitude decreases as the density $\rho$ increases ( $V$ decreases). The potential energy has a potential hill at $\chi=0$ and a valley at $\chi=1$. Beyond $\chi=1$, it increases monotonically. Besides the potential term there is another term proportional to $\chi^{2}$. As the density increases the $\mathcal{E}_{\sigma}$ contribution becomes relatively important. The thermal pion contribution is proportional to $T^{4} / \rho$. Thus, the term proportional to $\chi^{2}$ increases to overcome the potential hill.

Let us look into this phenomenon in detail. For a given $\rho$ and $T$, explicit dependence of eq.(44) on $\chi$ can be presented as

$$
\mathcal{E}=a \chi^{2}+b+c\left(\chi^{4}\left(\ln \chi-\frac{1}{4}\right)+\frac{1}{4}\right) .
$$

One may easily match the coefficients $a, b$ and $c$ with eq.(4). $\mathcal{E}$ has two local minima at $\chi=0$ (chirality symmetric phase) and at $\chi=\chi_{0}(\neq 0)$ (chirality broken phase) satisfying

$$
a+2 c \chi_{0}^{2} \ln \chi_{0}=0
$$

The energy difference $\Delta \mathcal{E} \equiv \mathcal{E}(\chi=0)-\mathcal{E}\left(\chi=\chi_{0}\right)$ is obtained as

$$
\Delta \mathcal{E}=\frac{1}{4} \chi_{0}^{2}\left(2 a-c \chi_{0}^{2}\right)
$$

The phase transition happens when $\Delta \mathcal{E}=0$; that is,

$$
2 a-c \chi_{0}^{2}=0
$$

Combining eqs.(6) and (8), we get $\chi_{0}$ at the critical point as

$$
\chi_{0}^{c}=e^{-1 / 4} .
$$

Note that the phase transition happens suddenly from a non-vanishing $\chi=\chi_{0}^{c}$ value to $\chi=0$. It is therefore a first order phase transition. However, this result is just a peculiarity of the used dilaton potential $\mathcal{V}(\chi)$. If we had taken $\mathcal{V}(\chi) \sim\left(\chi^{2}-1\right)^{2}$, the phase transition would have been second order. 


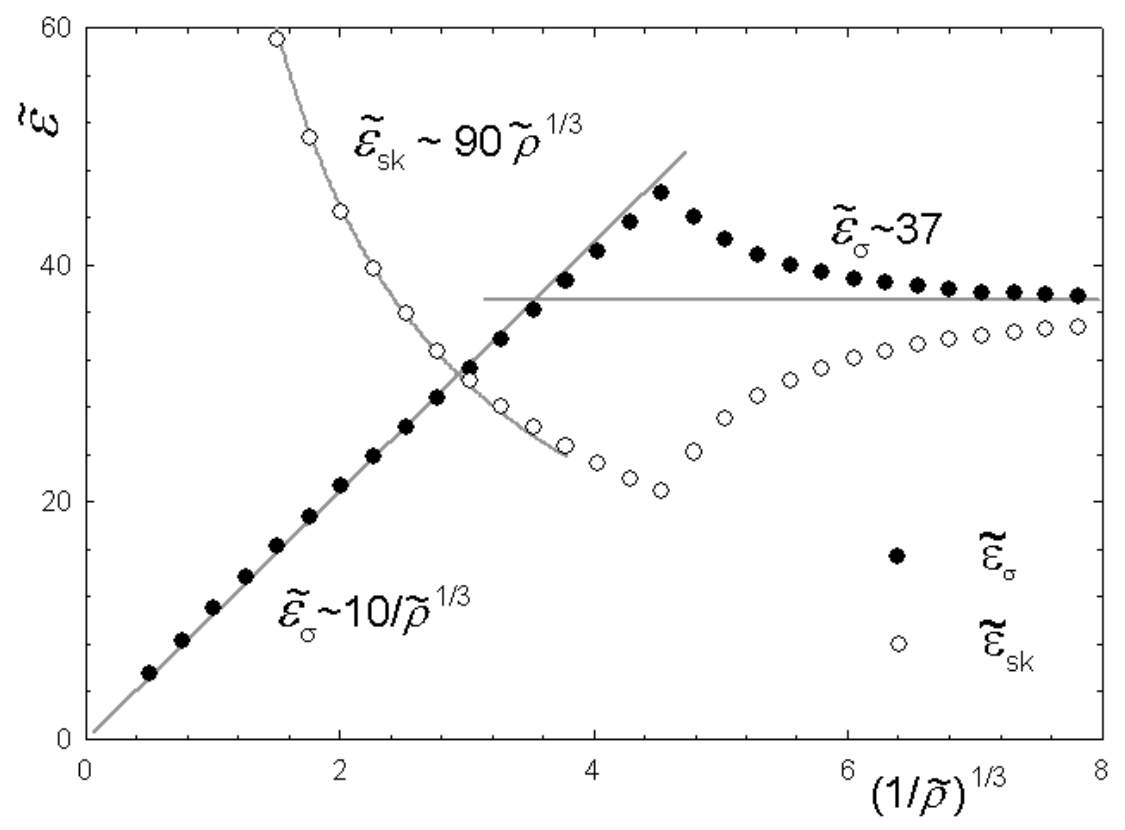

Figure 1: $\tilde{\mathcal{E}}_{\sigma}$ and $\tilde{\mathcal{E}}_{\text {sk }}$ as functions of $1 / \tilde{\rho}^{1 / 3}$.

Using the above equations at the critical point, we get

$$
\rho^{c} \mathcal{E}_{\sigma}+\frac{\pi^{2}}{10} T_{c}^{4}=\frac{f_{\chi}^{2} m_{\chi}^{2}}{8 e^{1 / 2}}
$$

which leads to

$$
T_{c}=\left(\frac{10}{\pi^{2}}\left(\frac{f_{\chi}^{2} m_{\chi}^{2}}{8 e^{1 / 2}}-\rho^{c} \mathcal{E}_{\sigma}\left(\rho^{c}\right)\right)\right)^{1 / 4}
$$

For $\rho=0$ (zero density), our estimate for the critical temperature is

$$
T_{c}=\left(\frac{10}{\pi^{2}} \frac{f_{\chi}^{2} m_{\chi}^{2}}{8 e^{1 / 2}}\right)^{1 / 4} \sim 205 \mathrm{MeV},
$$

where we have substituted $f_{\chi}=210 \mathrm{MeV}, m_{\chi}=720 \mathrm{MeV}$. It is remarkable that this naive toy model leads to $T_{c} \sim 200 \mathrm{MeV}$, which is quite close to that obtained by lattice QCD [2] and in agreement with the data [20].

To complete our study we compute $\mathcal{E}_{\sigma}$ as a function of $\rho$ by minimizing numerically $\mathcal{E}(\rho, T)$ with respect to $U_{0}(\vec{r})$ and $\chi$. An useful approximation may be obtained by fixing $\chi=1$ and minimizing $\mathcal{E}_{\sigma}(\rho)+\mathcal{E}_{\text {sk }}(\rho)$ with respect to $U_{0}(\vec{r})$. Since $\chi$ varies only in the range $e^{-1 / 4} \sim 0.8<$ $\chi<1$, before the system undergoes the phase transition, the approximation is quite reasonable. These minimum energy configurations can be found by various methods[21], and they turn out to be single skyrmion FCC (Face Centered Cubic) crystals at low density and half-skyrmion CC (Centered Cubic) crystals at higher density.

The dependence of $\mathcal{E}_{\sigma}$ and $\mathcal{E}_{\mathrm{sk}}$ on $f_{\pi}$ and $e_{\mathrm{sk}}$ can be separated out explicitly as

$$
\mathcal{E}_{\sigma, \mathrm{sk}}(\rho)=\left(f_{\pi} / e_{\mathrm{sk}}\right) \tilde{\mathcal{E}}_{\sigma, \mathrm{sk}}(\tilde{\rho}),
$$




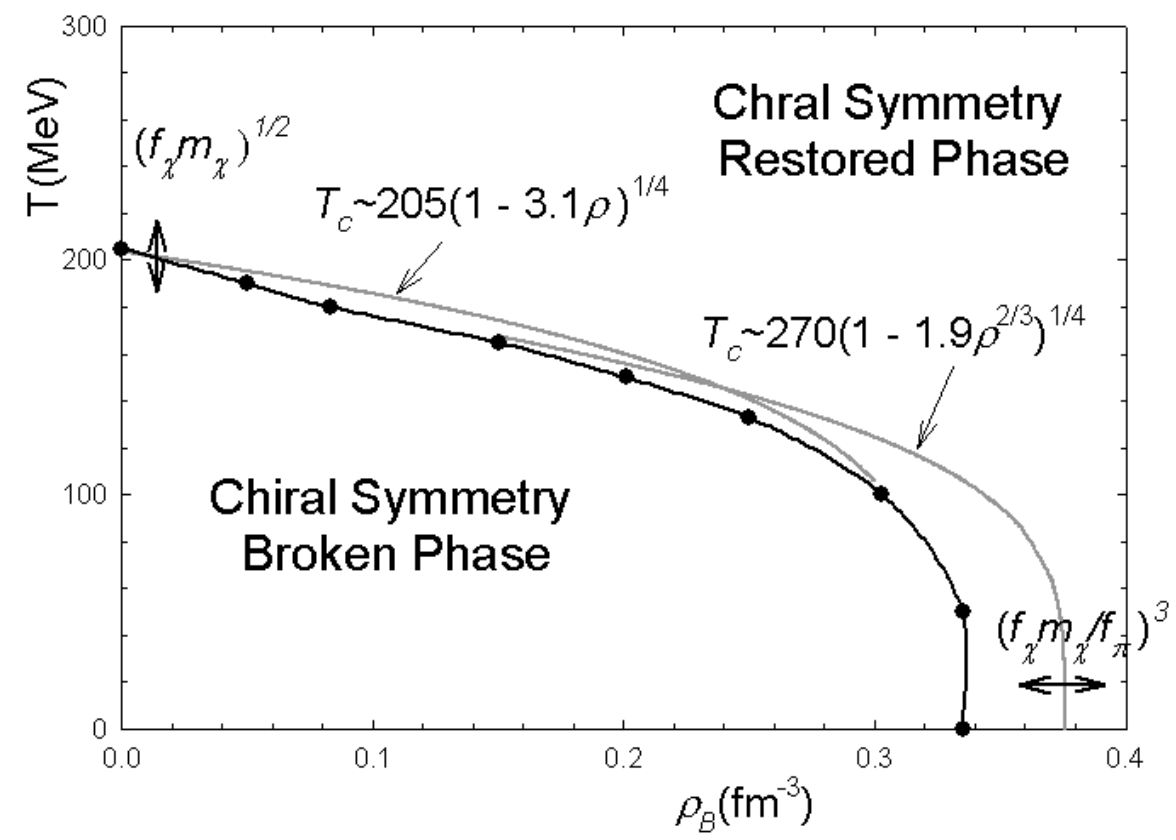

Figure 2: The chiral phase transition. The solid line shows the exact calculation, while the gray lines two approximate estimates.

where $\tilde{\rho}=\rho /\left(e_{\mathrm{sk}} f_{\pi}\right)^{3}$ and $\tilde{\mathcal{E}}_{\sigma, \mathrm{sk}}$ are dimensionless quantities which do not depend on $e_{\mathrm{sk}}$ and $f_{\pi}$. Shown in Fig. 1 are the numerical values on $\tilde{\mathcal{E}}_{\sigma}$ and $\tilde{\mathcal{E}}_{\mathrm{sk}}$. In order to show their dominant asymptotic behaviors, we present various values of $\tilde{\mathcal{E}}$ as a function of $1 /(\tilde{\rho})^{1 / 3}$. For high densities $\tilde{\mathcal{E}}_{\sigma}$ is linear in $1 /(\tilde{\rho})^{1 / 3}$, while for low densities it stays constant, implying that at a low density the interaction between skyrmions is negligible. We parameterize $\mathcal{E}_{\sigma}$ as

$$
\mathcal{E}_{\sigma}= \begin{cases}10 f_{\pi}^{2} / \rho^{1 / 3}, & \rho>\rho_{0} \\ 36 f_{\pi} / e_{\mathrm{sk}}, & \rho<\rho_{0},\end{cases}
$$

where $\rho_{0}=\left(e_{\mathrm{sk}} f_{\pi} / 3.6\right)^{3}$.

Using eq. (14) for $\mathcal{E}_{\sigma}$ and returning to eq. (10) we obtain the critical density for chiral symmetry restoration at zero temperature as

$$
\rho^{c}(T=0)=\left(\frac{f_{\chi}^{2} m_{\chi}^{2}}{8 e^{1 / 2}} \frac{1}{10 f_{\pi}^{2}}\right)^{3 / 2} \sim 0.37 \mathrm{fm}^{-3} .
$$

Since $\rho_{0}=0.24 \mathrm{fm}^{-3}<\rho^{c}(T=0)$ we have been consistent when using the high density formula for $\tilde{\mathcal{E}}_{\sigma}$

The resulting critical density $\rho^{c}(T=0) \sim 0.37 \mathrm{fm}^{-3}$ is only twice normal nuclear matter density and it is low with respect to the expected values. This result is not problematic since $\rho^{c}(T=0) \sim\left(f_{\chi} m_{\chi} / f_{\pi}\right)^{3}$ and $T_{c}^{\rho=0} \sim\left(f_{\chi} m_{\chi}\right)^{1 / 2}$ and therefore the description of the phase transition does not depend on the specific values of the parameters. Moreover, small changes in them may lead to larger values for the critical density.

For a finite density smaller than $\rho^{c}(T=0)$, we obtain the corresponding critical temperature by substituting the asymptotic formulas (14) for $\mathcal{E}_{\sigma}$, 


$$
\begin{aligned}
& T_{c}=T_{c}(\rho=0)\left(1-3.09\left(\mathrm{fm}^{3}\right) \rho_{c}\right)^{1 / 4} \quad \text { for } \rho<\rho_{0} \\
& T_{c}=T_{c}(\rho=0)\left(1-1.92\left(\mathrm{fm}^{2}\right) \rho_{c}^{2 / 3}\right)^{1 / 4} \text { for } \rho>\rho_{0}
\end{aligned}
$$

The results of these two curves are shown in Fig. 2 by gray lines.

The exact calculation (black dots connected by black line in Fig. 2) has been obtained numerically by minimizing the energy eq.(44). The resulting phase diagram has the same shape but the temperatures and densities are generally smaller than in the approximate estimates shown in Fig. 2.

There is a long history of success of the Skyrme model in describing the chiral symmetry restoration phase transition for dense hadronic matter [4, 5, 6, 7, 8, 9, 10]. The model might also be a viable description of the new phenomenology that is being proposed for dense/hot matter [22]. The time had come for an analysis of its behavior with temperature. We have presented a first description of the chiral restoration phase transition in the temperature-density plane, whose main ingredient is that the dominant mechanism is the absorption of heat by the fluctuating pions in the background of crystal skyrmion matter. This description leads to a phase transition whose dynamical structure is parameter independent and whose shape resembles much the conventional confinement/deconfiment phase transition. Moreover, for parameter values close the conventional ones, we obtain the expected critical temperatures and densities. Further investigation in these matters is required since it is becoming apparent that the path to the

phase transition is not without new physics and that this 'bottom up' approach might be useful to obtain interesting new physics [23].

\section{Acknowledgements}

Byung-Yoon Park was supported by a research fund from Chugnam National University, HeeJung Lee by the research grant of Chungbuk National University in 2008 and Vicente Vento by grant FPA2007-65748-C02-01 from Ministerio de Ciencia e Innovación.

\section{References}

[1] J. Phys. G 35 (2008) 104021 Quark Matter 2008

[2] For a review, see F. Karsch, Recent lattice results on finite temperature and dense QCD, part II, PoS (Lattice 2007) 015.

[3] M. Fromm and Ph. de Forcrand, arXiv:0811.1931 [hep-lat].

[4] H.-J. Lee, B.-Y. Park, D.-P. Min, M. Rho and V. Vento, Nucl.Phys. A723 (2003) 427.

[5] B.-Y. Park, M. Rho, V. Vento, Nucl. Phys. A736 (2003) 129.

[6] B.-Y. Park, M. Rho, V. Vento, Nucl. Phys. A807 (2008) 28.

[7] H.-J. Lee, B.-Y. Park, M. Rho and V. Vento, Nucl. Phys. A726 (2003) 69.

[8] H.-J. Lee, B.-Y. Park, M. Rho and V. Vento, Nucl. Phys. A741 (2004) 161.

[9] A. C. Kalloniatis and B.-Y. Park, Phys. Rev.D71(2005) 034010. 
[10] A. C. Kalloniatis, J. D. Carroll and B.-Y. Park, Phys. Rev. D71 (2005) 114001.

[11] I. Klebanov, Nucl. Phys. B 262 (1985) 133.

[12] A. S. Goldhaber, N. S. Manton, Phys. Lett. B 198231.

[13] See for example, A. D. Jackson, J. J. M. Verbaarschot, Nucl. Phys. A 501 (1988) 419.

[14] See, for example, M. Franz, Z. Tesanovic, O. Vafec, Phys. Rev. B66 (2002) 054536.

[15] G. Kälbermann, J. Phys. G 26 (2000) 129.

[16] O. Schwindt and N. R. Walet, arXiv:hep-ph/0201203.

[17] J. Ellis, J. Lanik, Phys. Lett. B 150 (1985) 289.

[18] G. E. Brown, M. Rho, Phys. Rev. Lett. 66 (1991) 2720.

[19] A. Bochkarev and J. Kapusta, hep-ph/9602405.

[20] Nucl. Phys. A 757 (2005) 1-283, First three years of operation of RHIC.

[21] M. Kugler, S. Shtrikman, Phys. Rev. D 40 (1989) 3421.

[22] L. McLerran and R. D. Pisarski, Nucl. Phys. A 796 (2007) 83 [arXiv:0706.2191 [hep-ph]].

[23] L. McLerran, arXiv:0808.1057 [hep-ph]. 